\title{
Reduction of Energy Hole in WSN
}

\author{
Jayant Kumar Rout
}

\begin{abstract}
Energy hole problem in the wireless sensor network (WSN) is a critical issue due to the energy discharge of the sensor nodes in a rapid manner which lies closer to the sink. This is because of the fact that nearer sensor nodes send their own information as well as the information received from other regions to the sink. After sometime these sensor nodes start losing their power levels and become incapable to transfer data to sink and perform other activities despite the fact the energy of nodes in other regions are still unused which further disturbs the network performance. In this article, we have proposed a Concentric Layered Hexagonal Network Division Approach (CLHND) for solving energy hole issue. Initially, the network is divided into concentric hexagons and each hexagon act as a different layer. After that, each hexagon is divided into six equal portions. In the subsequent stage, the larger layer will be selected from all other layers. Now to decrease additional energy discharging from this layer, numerous sensor nodes positioned. In the final phase to prevent the energy hole issue, a suitable directing and ordering have been done which further improves network lifetime. The simulation results showed that the proposed CLHND approach has resolved the energy hole issue as compared to the existing techniques such as HRTBR and SEHP.

Keywords : WSN, Clustering, Energy hole, Hexagon Network, Layered approach.
\end{abstract}

\section{INTRODUCTION}

T he sensor network is a collection of a huge amount of sensor nodes which are equipped with sensing and communication capabilities[1]. The sensor nodes are battery powered so the replacement of these nodes is a tough task[2]. To augment the network lifespan, energy of the nodes in the WSN should be utilized in an efficient way. The nodes in a wireless sensor network (WSN) works together to form a complaisant network to monitor the region or environment[3]-[5]. WSN works as a powerful application to observe any phenomenon or any condition. Practically, the sensor network are used in several situations and modalities such as thermal[6], [7], acoustic and radar depending upon the nature of application. The various areas in which WSN applications are used nowadays include military, weather, environment monitoring, healthcare etc. In the battlefield the WSN is used to track the positions of the soldiers and tanks. The nodes in the wireless networks communication their information to each other over the wireless links and collectively pass the data to the base station (BS) for the processing of data. A proficient energy utilization scheme in the WSN can improve the performance and lifespan of the network[2].

Revised Manuscript Received on December 30, 2019.

* Correspondence Author

Jayant Kumar Rout*, Department of Electronics and Communication Engg., ITER, Siksha 'O' Anusandhan (Deemed to be University), Bhubaneswar, India. Email: jayantrout@soa.ac.in

(C) The Authors. Published by Blue Eyes Intelligence Engineering and Sciences Publication (BEIESP). This is an open access article under the CC BY-NC-ND license (http://creativecommons.org/licenses/by-nc-nd/4.0/)
The lifespan of the network in the WSN is considered as one of the major issues[2]. For enhancing the lifespan of network various approaches are used[8]-[10]. But in few networks there exist a problem of the energy hole in WSN.

The energy hole problem is a vital concern in the WSN. Sensor nodes that lie closer to the sink lose their energy in a rapid manner as contrasted to the nodes which are far-flung from the sink. Hence lifetime of those sensor nodes is less which further reduces the lifetime of the over-all network. This singularity of irregular energy consumption degrees is named as energy hole issue[11][12][13]that may outcome quick dysfunction of the whole network, although other portions of the system still have loads of energy[14].

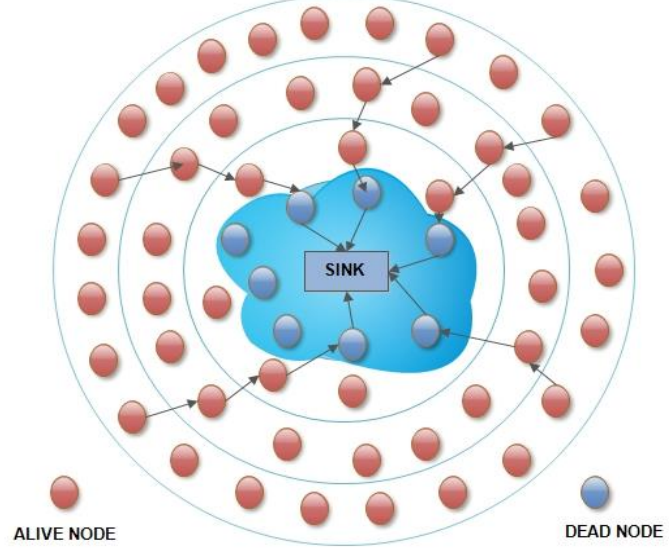

Fig.1 Energy Hole issue in WSN

Figure 1 reflects that nodes in the blue region have died because they are nearer to the sink and discharging their energy in rapid fashion. In the beginning, energy hole issue for the homogeneously distributed network is identified by authors in [15][16] for which region is separated into the concentric haloes from the sink. Simulation outcomes display that, as soon as the first halo disbursed all its energy, the consumption of energy by a sensor node in the $10^{\text {th }}$ halo is very less as compared to those which are closer to sink. Authors in [17] examine the issue of irregular energy consumption in an outsized course of many to one sensor systems[18]. They detected that, ordered positioning and traffic flow density could have optimistic belongings on the issue, although weighty sensor node compactness or extra data generations have petite or unwanted special effects on it. Non- uniform circulation technique[19][20] is offered to resolve energy hole issue. It has been revealed that uneven dissemination technique can deliver a composed energy ingesting ratio of the nodes. Moreover, energy hole issue in the systems one or the other cannot relate unswervingly to the sink for the reason that of inadequate radio range or unswerving distribution of the data will bound energy and diminish over-all network lifetime[21].

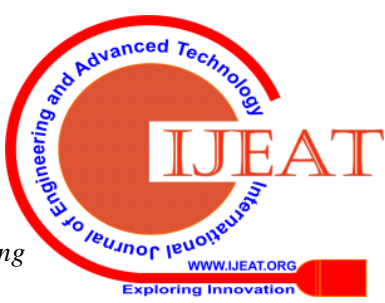




\section{Reduction of Energy Hole in WSN}

\section{RELATED WORK}

The sensor nodes that are nearby sink will come across by way of energy fall by growing traffic flow of nodes. As soon as sensor nodes do not work properly, the over-all network functioning gets stopped and hence this procedure can cause primary death in the network even if energy still remains in some of the sensor nodes, the system will not work fine[22]. Authors in[20]for resolving the energy hole issue, deployed a number of sensor nodes nearby the sink. Authors in [23]for resolving energy hole issue, used two sensor nodes with additional energy deployed in the central region of the system. Sensor nodes that are nearby to sink will transfer data straight away to the BS once the clustering is done. The nodes that are far-off from BS will send data to these two sensor nodes. As these nodes have reasonably high energy contrasted to the other nodes in the system, because of which the network generally does not fail, since these nodes work as a bond. Nearby sensor nodes which are positioned in radio range does not consume additional energy. Therefore energy hole issue can be prohibited in the network.

In [24] author proposed an approach for solving energy hole issue, in which energy consumption in the cluster as well as in Cluster head $(\mathrm{CH})$ was very less. In this technique, the cluster is not identical and circulation of nodes is non-uniform in the network. Authors in[25][26]proposed a new algorithm based on the multi-level concept and separating levels in two parts. In the first part, $\mathrm{CH}$ send the data to BS and in the succeeding steps Gauss elimination process is used for reducing energy consumption in the system but one of the main problems with this method is that selection of $\mathrm{CH}$ is done by BS. In some papers authors proposed a new algorithm HRTBR for which they construct the rounded network and implement hierarchy synthetic routing. Simulation results display that energy hole issue has been reduced up to a certain amount. The author recommends a new type of method to solve energy hole issue, that uses generally mobile sensor to restore energy holes at the rate of very high cost. Authors in [24]recommend an uneven node circulation plan that can accomplish a sub-stable energy ingesting of the sensor nodes.

In [21] the author proposed a technique to solve the energy hole issue. The area is divided into concentric circles. This approach has reduced the problem to some extend.

\section{ENERGY MODEL FOR WSN}

The energy consumption in WSN is the summation of the aggregate energy disbursed by all the nodes in the system. The energy usage in the WSN plays a substantial role in defining the lifespan of the network. The model of energy shows the process of energy usage in the system in sending or receiving data. Figure 2 portrays the model of energy in which $\mathrm{k}$ bits data is being transferred amongst two segments of energy model which are d distance apart.

The formula for the receive and transmit of data packets in WSN is given by the equations below:

$$
E_{t r}(k, d)= \begin{cases}k E_{\text {elec }}+k \varepsilon_{f s} d^{2} & d<d_{0} \\ k E_{\text {elec }}+k \varepsilon_{\text {amp }} d^{4} & d \geq d_{0}\end{cases}
$$
amplification data

- $\quad E_{\text {elsc }}$ is the energy spent to transfer a single data bit

- $\varepsilon_{f s}$ and $\varepsilon_{a m p}$ represents the coefficient of

- $E_{D A}$ represents the energy used in aggregation of

- $E_{T X}$ denotes the energy of transmission $E_{R X}$ is energy of reception of $k$ data bits and $d$ is the separation among receiver and transmitter.

\section{PROPOSED CLHND MODEL}

The major constraint of WSN is the lifespan of the network. If the energy usage in the network can be managed properly then the life of the network can be enhanced. Sensor nodes transmit information received from other sensor nodes of the system to the BS. Afterward, a small span of time the nodes which are nearby to the sink starts releasing their energy in a rapid way since these sensor nodes are continuously transferring the data to the BS as well as to the other nodes within the network. As a result, energy hole issue arises in the network and over-all network become dead only because of the death of some sensor nodes. We have proposed a Concentric Layered Hexagonal Network Division Approach (CLHND) in order to avoid the uneven energy consumption of the nodes which are closer to the sink. These are some steps that have been used in this proposed algorithm which further reduces the energy hole issue up to the significant amount. Initially, the network is divided into a concentric hexagon and each hexagon act as a different layer.

Also, each hexagon is divided into six equal portions. In the subsequent stage, the larger layer will be selected from all other layers. Now to decrease additional energy discharging from this layer, several sensor nodes are positioned. In the final phase to prevent the energy hole issue, a suitable directing and ordering have been done which further improves network lifetime. Selection of $\mathrm{CH}$ is done in terms of node's remaining energy as well the node distance.

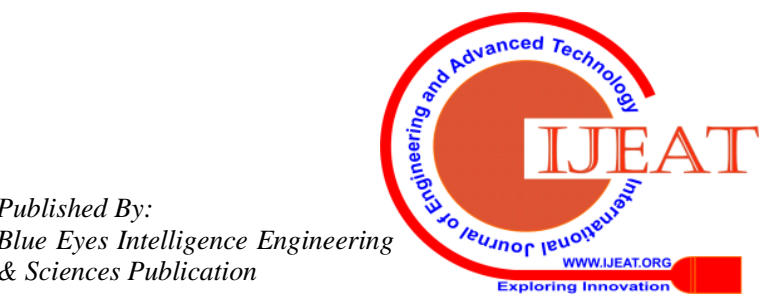


It is supposed in the proposed algorithm that the sensor nodes are dispersed haphazardly in the first layer. Once the network formation is done, the sensor nodes with the help of GPS send their features and ID to the sink. Once they send their features and ID to the sink, sensors turn off their GPS to save their energy. After getting signals by BS the table will be updated automatically with new characteristics and ID of different nodes. Now clustering is completed in most of the layers and suitable node for transferring data to the lower layer is selected by using this info.

The last Cluster head transmits this data to the nodes of the $1^{\text {st }}$ layer by bearing in mind closeness and residual energy of the $1^{\text {st }}$ layer sensor nodes. After getting info and assembly their data, $1^{\text {st }}$ layer sensor nodes will transfer their data straight away to the sink. As there are many nodes in the $1^{\text {st }}$ layer and inappropriate range, which finally removes the energy hole issue in the system. Figure2 represents the proposed CLHND algorithm where it can be clearly seen that the nodes have been distributed near the sink to overcome the energy hole issue.

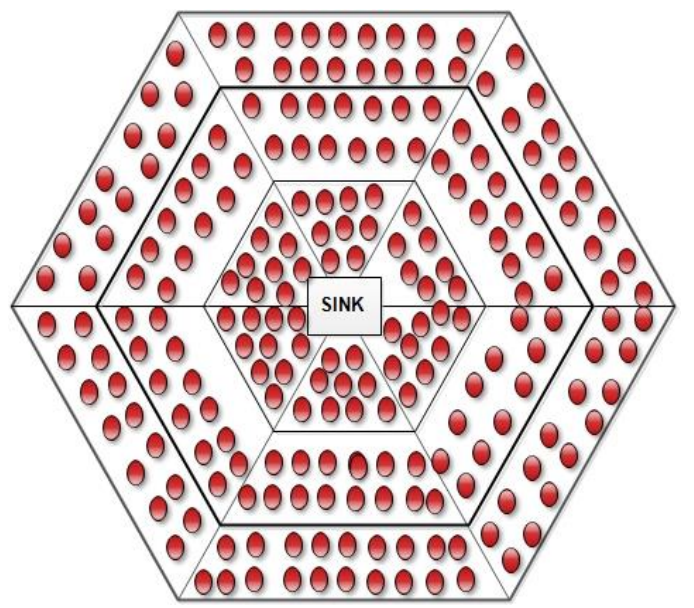

Fig.3 Proposed CLHND Algorithm

Following steps have been taken into consideration for designing proposed CLHND algorithm.

Step1: Surroundings are measured in area and measurement.

Step2: BS is retained in the midpoint of the network for relaxed retrieving to sensor nodes and small energy feeding.

Step3: Split measured surroundings by concentric hexagon as seen in Figure1

Step4: Layers is achieved by separating furthest sensor node to BS.

Step5: Layer is separated in six identical portions of the hexagon.

Step6: Selection of $\mathrm{CH}$ head is done in all the layers except $1^{\text {st }}$ layer based on remaining energy and distance of sensor node.

For the selection of $\mathrm{CH}$ between ' $\mathrm{n}$ ' numbers of nodes average distance of each node with another node from the same cluster is achieved by equation (1) which is given below

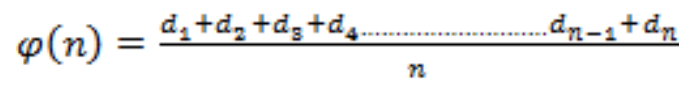

(3)

Where $d_{i}$ is the distance between any two neighboring node which is calculated by Euclidian formula given in equation (2)

$$
d_{i}=\sqrt{\left(x_{i}-x_{j}\right)^{2}+\left(y_{i}-y_{j}\right)^{2}}
$$

Where $\left(x_{i}, y_{i}\right)$ and $\left(x_{j}, y_{j}\right)$ are the co-ordinates of the nodes. In the case where we have n number of nodes in the n-dimensional space then we can define Euclidean distance matrix which is given by:

$$
A=\left[\begin{array}{cccc}
0 & d_{12}^{2} & \cdots & d_{1 n}^{2} \\
d_{21}^{2} & 0 & \cdots & d_{2 n}^{2} \\
\vdots & & & \vdots \\
d_{n 1}^{2} & d_{n 1}^{2} & \cdots & 0
\end{array}\right]
$$

So the probability of any node to become $\mathrm{CH}$ is given by equation (5)

$$
P=\frac{E_{n}}{\varphi(n)}
$$

Where, $E_{n}$ is the node's residual energy.

In this procedure, certain sensor nodes are designated as a $\mathrm{CH}$. Further on the basis of TDMA schedule, $\mathrm{CH}$ assigns a specific time to their member nodes to transmit their data to the $\mathrm{CH}$. Once the data is gathered by $\mathrm{CH}$, the gathered data is sent to the lower layer $\mathrm{CH}$ and a $\mathrm{CH}$ is designated again for lower level clusters [6]. $3^{\text {rd }}$ layer $\mathrm{CH}$ after assembly the data from $3^{\text {rd }}$ layer transfer data to $2^{\text {nd }}$ layer. $\mathrm{CH}$ of $2^{\text {nd }}$ layer accumulates data of $2^{\text {nd }}$ and $3^{\text {rd }}$ layer. Again Selection of $\mathrm{CH}$ according to the residual energy as well as the distance of that node and hence data transported to that node. Sensor nodes of $1^{\text {st }}$ layer direct data to BS and also different nodes of $1^{\text {st }}$ layer transmit their specific data as shown in Figure4.

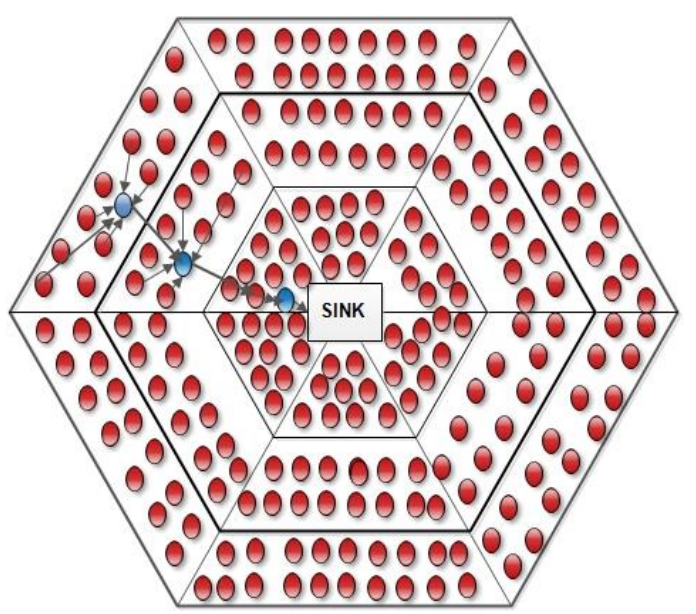

Fig.4 Flow of data in Proposed Algorithm

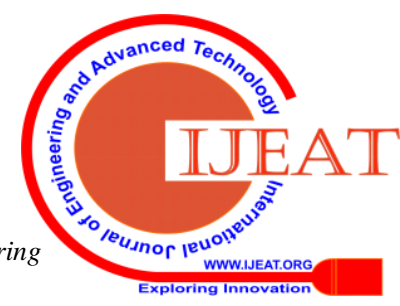




\section{Reduction of Energy Hole in WSN}

\section{SIMULATION AND RESULTS}

Simulation of proposed algorithm CLHND has been done using MATLAB and the comparison is performed with existing algorithm HRTBR and SEHP to measure the effectiveness of the proposed work. Various parameters have been analyzed during simulation like delivery ratio, the average remaining energy of nodes and the number of the lost packet. Simulation variable and its value is listed in Table 1.

Table 1: Simulation Variables

\begin{tabular}{|l|l|}
\hline Simulation Variable & Set up values \\
\hline Network area & $150 \mathrm{~m} \times 150 \mathrm{~m}$ \\
\hline No. of sensor nodes & 200 nodes \\
\hline Preliminary energy & $0.5 \mathrm{~J}$ \\
\hline Sink location & $(50 \mathrm{~m}, 150 \mathrm{~m})$ \\
\hline Simulation Round & 5000 \\
\hline
\end{tabular}

Table 2 shows the parameters taken during the simulation. The value of these parameters is used to perform the comparison between the proposed technique and the previous approaches.

Table 2: Simulation Parameters

\begin{tabular}{|l|l|}
\hline Simulation Parameters & Values \\
\hline$\varepsilon_{a m p}$ & $0.0011 \mathrm{pJ} / \mathrm{bit} / \mathrm{m}^{4}$ \\
\hline $\boldsymbol{E}_{\text {elec }}$ & $15 \mathrm{~nJ} / \mathrm{bit}$ \\
\hline $\boldsymbol{E}_{D A}$ & $6 \mathrm{~nJ} / \mathrm{bit}$ \\
\hline $\boldsymbol{\varepsilon}_{f \mathrm{~s}}$ & $9 \mathrm{pJ} / \mathrm{bit} / \mathrm{m}^{2}$ \\
\hline $\boldsymbol{d}_{0}$ & $100 \mathrm{~m}$ \\
\hline $\boldsymbol{M}_{\min }$ & 0.3 \\
\hline
\end{tabular}

Figure5 shows the plot of packet delivery rate. Simulation outcome reflects that packet delivery rate is more in proposed CLHND algorithm compared to the existing algorithm. This is only because of consumption of energy in the first layer is less compared to the existing HRTBR and SEHP algorithm. Also when packet generation amount rises in the system then data delivery amount rate keeps reducing.

Average of residual energy with the influence of packet generation amount rate and with the influence of node's initial energy have been shown in Figure6 and Figure7 respectively. Simulation results show that sensor nodes residual energy in proposed CLHND technique is more as contrasted to the HRTBR and SEHP algorithm. In HRTBR and SEHP algorithm, every single node and subsequent sensor nodes interchange control messages by means of query message assured of the existence of afterward sensor nodes in a detailed period.

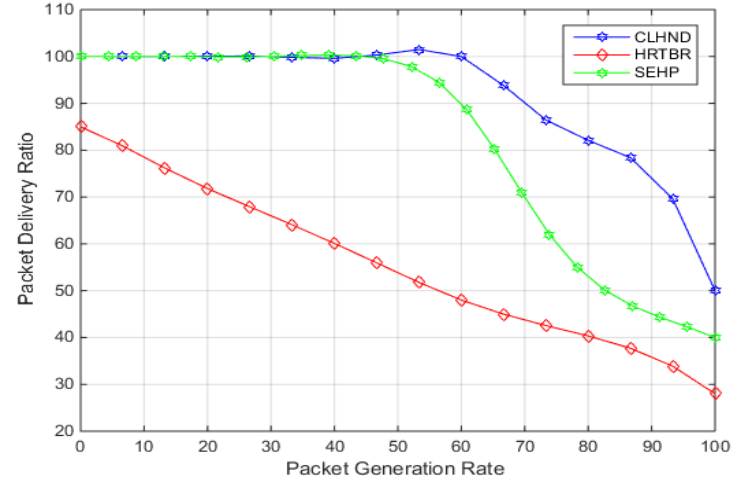

Fig.5 Packet Delivery Ratio

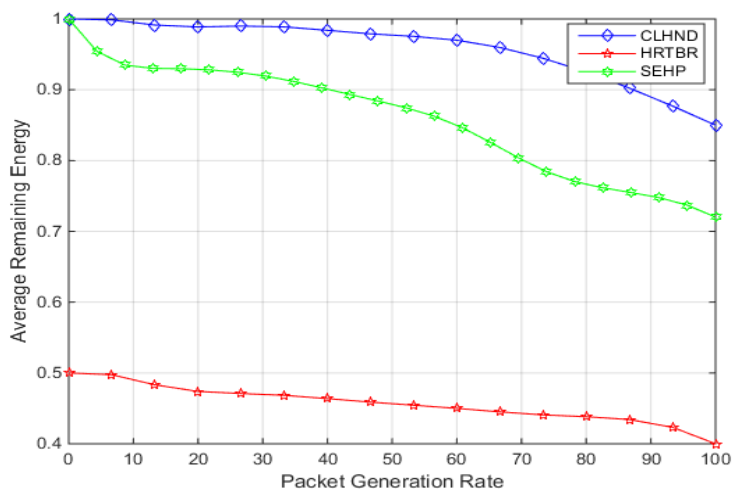

Fig.6 Average of remaining energy with the influence of packet generation rate

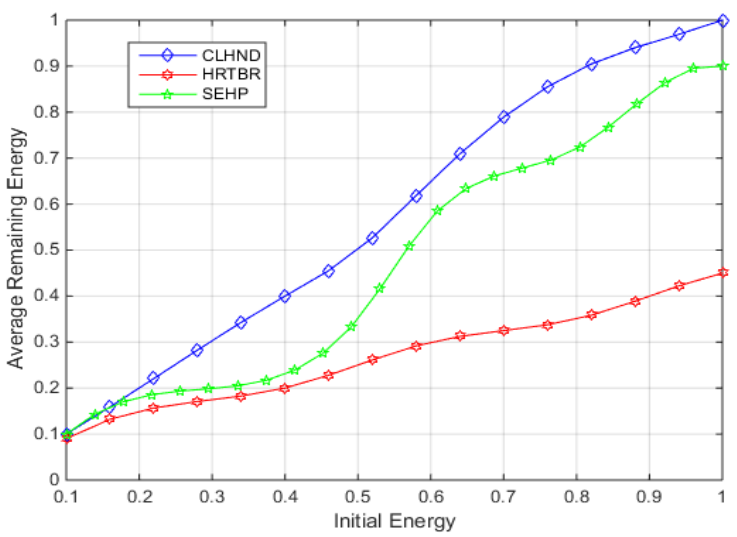

Fig.7 Average of remaining energy with the influence of nodes initial energy

Figure 6 and Figure 7 portrays that the proposed CLHND protocol has enhanced the performance of system as compared to the existing HRTBR and SEHP protocols. In case of proposed protocol the average remaining energy is more which causes the system to remain alive for longer amount of time.

Figure8 shows the lost packet in the network. Results of simulation shows that the number of the lost packet is less in proposed algorithm as compared to the existing algorithm. This is only happening in the network because the data delivery rate is more in the proposed algorithm. Consequently, by reason of growing data delivery rate in the system, most of the generated packet is transmitted to the sink.

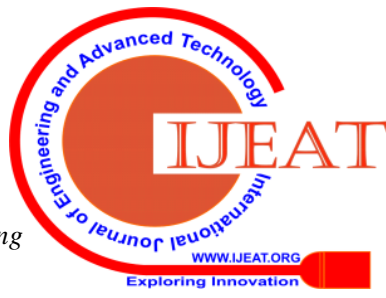


It is also clearly seen from the graph that when packet generation rate increases then lost number of the packet in the network are almost nearer to each other this is only happening for the reason that of when data generation rate rises in HRTBR algorithm then alive nodes decrease in the network.

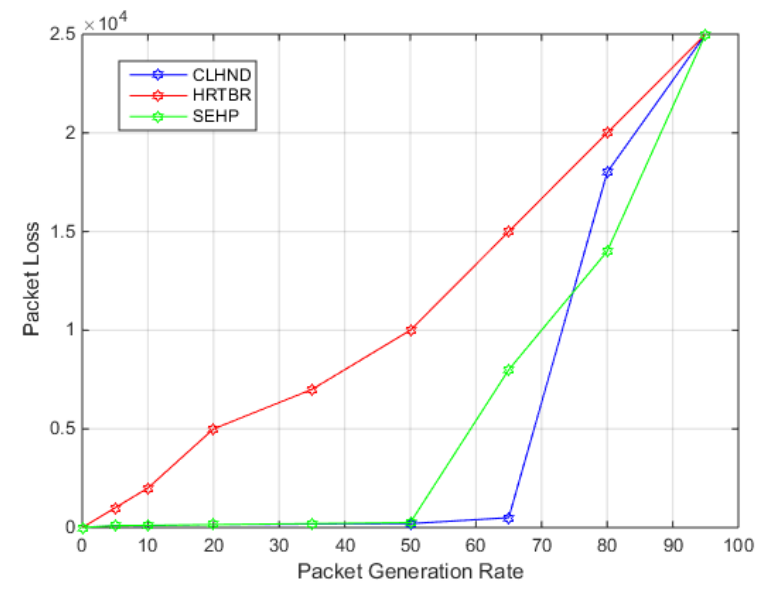

Fig.8 Lost Packet

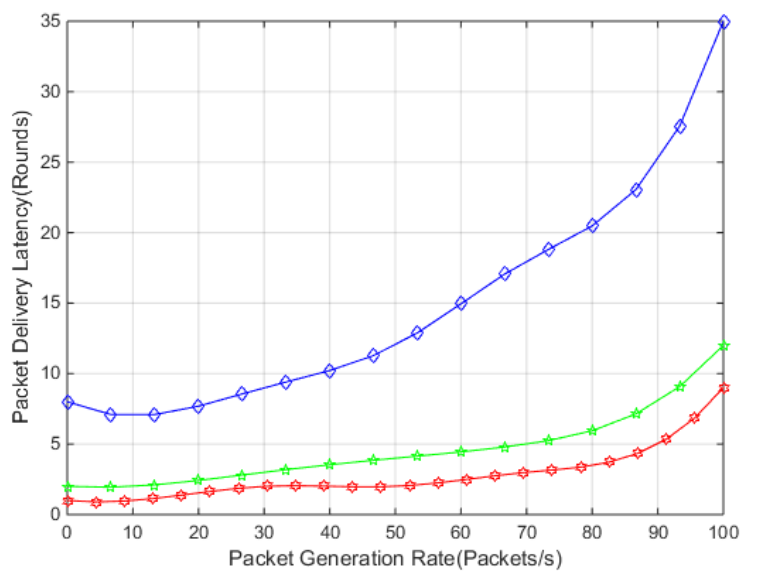

Fig.9 Packet Delivery Latency

Figure 9 shows the plot between the packet generation rate vs the packet delivery latency. From the figure it can be clearly portrayed that the latency in case of proposed technique is less as contrasted to the HRTBR and SEHP protocols. This shows that the proposed CLHND approach gas enhanced the performance of the system in many aspects and has reduced the issue of energy hole in the network.

\section{CONCLUSION}

In this paper, a new algorithm namely CLHND has been proposed and simulated using MATLAB simulator to overcome energy hole issue in the network. Network performance has been measured in terms of various parameters like delivery ratio, the average remaining energy of nodes and the number of the lost packet in the network. Simulation results reflect that proposed CLHND algorithm is performing well in all the parameters compared to the existing HRTBR and SEHP protocol. Further results may be enhanced with a different kind of concentric structure and with the division of network in different layers.

\section{REFERENCES}

1. I. F. Akyildiz, W. Su, Y. Sankarasubramaniam, and E. Cayirci, "A survey on sensor networks," IEEE Commun. Mag., vol. 40, no. 8, pp. 102-105, 2002.

2. C. F. García-hernández, P. H. Ibargüengoytia-gonzalez, J. Garcia-hernandez, and J. a Perez-diaz, "Wireless Sensor Networks and Applications : a Survey," J. Comput. Sci., vol. 7, no. 3, pp. 264-273, 2007.

3. R. Priyadarshi, L. Singh, A. Singh and A. Thakur, "SEEN: Stable Energy Efficient Network for Wireless Sensor Network," 2018 5th International Conference on Signal Processing and Integrated Networks (SPIN), Noida, Delhi-NCR, India, 2018, pp. 338-342. doi: 10.1109/SPIN.2018.8474228

4. R. Priyadarshi, L. Singh, Randheer and A. Singh, "A Novel HEED Protocol for Wireless Sensor Networks," 2018 5th International Conference on Signal Processing and Integrated Networks (SPIN), Noida, Delhi-NCR, India, 2018, pp. 296-300. doi: 10.1109/SPIN.2018.8474286

5. A. F. Liu, X. Y. Wu, Z. G. Chen, and W. H. Gui, "Research on the energy hole problem based on unequal cluster-radius for wireless sensor networks," Comput. Commun., vol. 33, no. 3, pp. 302-321, 2010.

6. W. B. Heinzelman, "Application-Specific Protocol Architectures for Wireless Networks," MASSACHUSETTS Inst. Technol., no. Ph. D Dissertation, pp. 1-154, 2000.

7. S. Olariu and I. Stojmenović, "Design guidelines for maximizing lifetime and avoiding energy holes in sensor networks with uniform distribution and uniform reporting," in Proceedings - IEEE INFOCOM, 2006.

8. Priyadarshi, R., Rawat, P., \& Nath, V. (2018). Energy dependent cluster formation in heterogeneous wireless sensor network.Microsystem Technologies. doi:10.1007/s00542-018-4116-7

9. Priyadarshi, R., Soni, S. K., \& Nath, V. (2018). Energy efficient cluster head formation in wireless sensor network. Microsystem Technologies, 24(12), 4775-4784. doi:10.1007/s00542-018-3873-7

10. A. Demertzis and K. Oikonomou, "Avoiding energy holes in wireless sensor networks with non-uniform energy distribution," in IISA 2014 5th International Conference on Information, Intelligence, Systems and Applications, 2014, pp. 138-143.

11. J. Li and P. Mohapatra, "An analytical model for the energy hole problem in many-to-one sensor networks," in IEEE Vehicular Technology Conference, 2005, vol. 4, pp. 2721-2725.

12. J. Li and P. Mohapatra, "Analytical modeling and mitigation techniques for the energy hole problem in sensor networks," Pervasive Mob. Comput., vol. 3, no. 3, pp. 233-254, 2007.

13. X. Wu, G. Chen, and S. K. Das, "On the energy hole problem of nonuniform node distribution in wireless sensor networks," in 2006 IEEE International Conference on Mobile Ad Hoc and Sensor Systems, MASS, 2006, vol. 1, pp. 180-187.

14. Priyadarshi, R., Soni, S. K., \& Sharma, P. (2019). An enhanced GEAR protocol for wireless sensor networks.In Lecture Notes in Electrical Engineering. doi:10.1007/978-981-13-0776-8_27.

15. R. Priyadarshi, H. Tripathi, A. Bhardwaj, A. Thakur, and A. Thakur, "Performance metric analysis of modified LEACH routing protocol in wireless sensor network," Int. J. Eng. Technol., vol. 7, no. 1-5, p. 196, Dec. 2017.

16. R. Priyadarshi, L. Singh, S. Kumar, and I. Sharma, "A hexagonal network division approach for reducing energy hole issue in WSN,"Eur. J. Pure Appl. Math., vol. 118, 2018.

17. J. Lian, K. Naik, and G. B. Agnew, "Data capacity improvement of wireless sensor networks using non-uniform sensor distribution," Int. J. Distrib. Sens. Networks, vol. 2, no. 2, pp. 121-145, 2006

18. J. N. Al-Karaki and A. E. Kamal, "Routing techniques in wireless sensor networks: a survey," IEEE Wirel. Commun., vol. 11, no. 6, pp. 6-28, Dec. 2004.

19. H. Karl and A. Willig, Protocols and Architectures for Wireless Sensor Networks. 2006.

20. H. M. Ammari, "Investigating the energy sink-hole problem in connected k -Covered wireless sensor networks," IEEE Trans. Comput., vol. 63, no. 11, pp. 2729-2742, 2014.

21. G. Ma and Z. Tao, "A nonuniform sensor distribution strategy for avoiding energy holes in wireless sensor networks," Int. J. Distrib. Sens. Networks, vol. 2013, 2013.

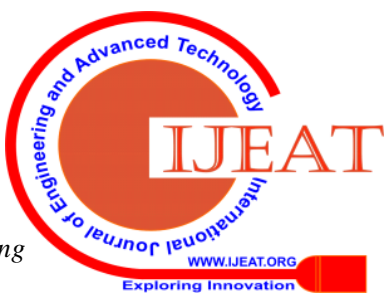




\section{Reduction of Energy Hole in WSN}

22. S. A. Nikolidakis, D. Kandris, D. D. Vergados, and C. Douligeris, "Energy efficient routing in wireless sensor networks through balanced clustering," Algorithms, vol. 6, no. 1, pp. 29-42, 2013.

23. R. E. Mohemed, A. I. Saleh, M. Abdelrazzak, and A. S. Samra, "Energy-efficient routing protocols for solving energy hole problem in wireless sensor networks," Comput. Networks, vol. 114, pp. 51-66, 2017.

24. Y. Liu, W. Dai, K. Xu, and M. Zheng, "A hybrid routing tree to avoid the energy hole problem in wireless sensor network," in Advances in Intelligent and Soft Computing, 2012, vol. 143 AISC, pp. 869-876.

25. H. Shiue, G. Yu, J. Sheu, S. Hung-Yu, Y. Gwo-Jong, and S. Jang-Ping, "Energy Hole Healing Protocol for Surveillance Sensor Networks," Was. Work. Wireless, Ad Hoc, Sens. Networks, 2005.

26. W. B. Heinzelman, A. P. Chandrakasan, and H. Balakrishnan, "An application-specific protocol architecture for wireless microsensor networks," IEEE Trans. Wirel. Commun., vol. 1, no. 4, pp. 660-670, 2002.

\section{AUTHORS PROFILE}

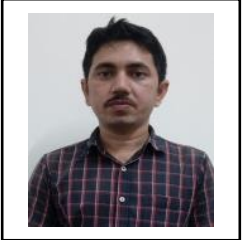

Jayant Kumar Rout received his BTech

in Electronics and Communication

Engineering from Utkal University, Odisha,

India in 2003. His research interest includes

Ultra-Wide Band Communication, Wireless

Sensor Network, Software Defined Radio and

Internet of Things.

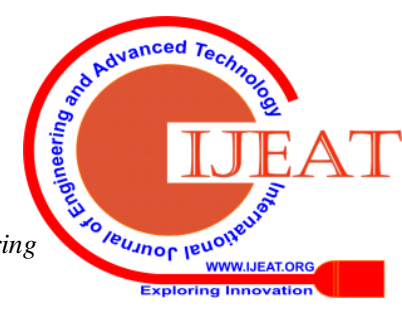

